\title{
EDITORIAL
}

\section{Molecular psychiatry: does a new field need to examine strategy?}

In clinical psychiatry one soon learns to look for patterns that repeat themselves over time because they reveal important themes. As we start our second volume we can start to identify themes that are emerging within molecular psychiatry. These include genetics, molecular neurobiology, histological methods applied to brain tissue, imaging, and the very recent advances in the molecular neurobiology of Alzheimer's disease. Within these broad themes, there are specific points that deserve attention. In my view these include association studies, the issue of using postmortem tissue in research studies, and whether recent progress in Alzheimer's disease will be soon paralleled by progress on the fundamental biological mechanisms of other psychiatric disorders.

The issues related to postmortem work and recent advances in research on Alzheimer's disease have been covered by recent articles in Molecular Psychiatry. ${ }^{1-4}$ I will make a few personal comments on association studies. Our field needs to come to a consensus on the meaning of statistically significant (or non-significant) associations. Let's assume that $60 \%$ of individual s with a common, complex psychiatric disorder have a specific polymorphism in a gene that might be relevant to the disease, in contrast to an overall incidence of $35 \%$ for that same polymorphism in the general population, with a $\mathrm{P}$ of $<10^{-5}$. What is the value of such a finding? Does it bring us closer to the cause of the disease in question? Does it tell us that a specific gene is indeed involved in the pathophysiology of the disorder? Does it facilitate diagnosis? Does it influence treatment? What happens when other investigators, studying other populations, find different rates of incidence in patients and controls for that specific polymorphism? The reverse is also an important point. What should one do when one paper reports lack of association between a specific gene and a disease? Should others continue to pursue that association? If a positive finding does come out, how does one integrate it with previous negative reports? Interestingly, some in this field seem to generate mostly negative data, while some others generate a fair amount of positive data. Is that related to personal differences in how one approaches research? These important questions deserve discussion because the number of association studies will

Correspondence: J Licinio, MD, Unit on Clinical Research, Clinical Neuroendocrinology Branch, Intramural Research Program, National Institute of Mental Health, NIH, BIdg 10, Rm 3S231, Bethesda, MD 20892-1284, USA. E-mail: licinio@nih.gov Received 8 November 1996; revised and accepted 13 November 1996 continue to grow as the full integration of molecular medicine and clinical psychiatry moves forward. ${ }^{5}$ Please advise:<licinio@nih.gov>.

Molecular biology offers much that is exciting for us in psychiatry. Using the tools of molecular medicine it is now possible to rapidly move from bench to bedside and back in molecular psychiatry. Psychiatric disorders are characterized by disordered function of the central nervous system (CNS), resulting in many types of alterations, particularly the processing of information. Signal processing is a key function of the brain: we can conceptualize molecular psychiatry as a field that studies signaling, at the most fundamental intracellular level as well as at the extracellular level. Signal transduction is affected by the genome and by the environment. The end results of intra- and extracellular signaling are functions such as mood, cognition, and behavior. Conceptual izing molecular psychiatry as a discipline that studies CNS functions that are affected by the processing of intra- and extracellular signals, we can keep a focus as we repeatedly move between bench and bedside (see Figure 1).

These are not original ideas. The integration of internal medicine and molecular biology started in the 1980s. As that happened many investigators in fields such as molecular endocrinology questioned whether prior approaches such as hormone measurements were still needed in clinical research. The conclusions of the discussions that took place in the field of endocrinology are that organized signaling occurs at every level, from the microcosm where one structure interlocks with another at high affinity - affecting how long they are in a suitable conformation, to second messengers and phosphorylated proteins, and to regulated timing in fairly complex feedback loops involving several chemical messengers interacting in the blood stream. Intra- and extra-cellular signaling are parts of a homeostatic network which is crucial for the optimal func-

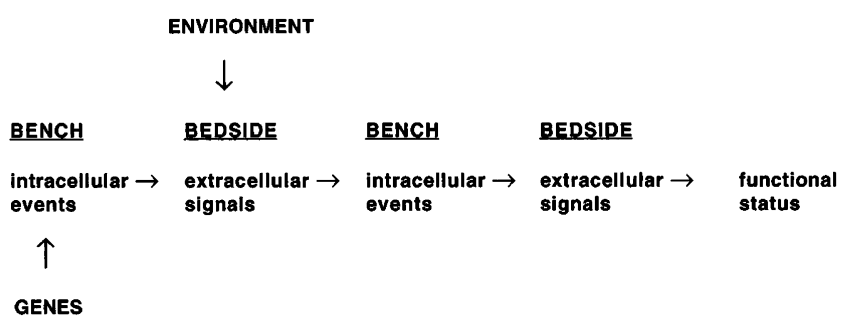

Figure 1 Defining molecular psychiatry as a field that studies functions that are affected by signaling at the intracellular and extracellular levels makes it possi ble to keep a conceptual focus during transitions from bench to bedside. 
tioning of adaptive mechanisms that have to rapidly respond to changes in internal milieu and in the environment: Those adaptations occur minute to minute and hour to hour. Disease is a dysruption in these rapidly changing adaptive mechanisms. The discussions that took place in endocrinology as molecular endocrinology interfaced with clinical research in the 1980s have led to refinements in clinical research strategies that make it possible to examine how al terations in signaling at the clinical and molecular levels contribute to disease. ${ }^{6,7}$ These are elegantly reviewed by JD Veldhuis in this issue of Molecular Psychiatry. ${ }^{7}$ It is therefore unnecessary for us in molecular psychiatry to spend much time repeating questions that have been so thoughtfully addressed by other disciplines. A conceptual framework to move from bench to bedside examining the processing of signals at the intra- and extracellular levels has al ready existed for several years. It is now time to change our focus from the intellectually exciting task of examining strategy to the more important mission of testing specific research hypotheses that elucidate key points in biology.

J Licinio, MD

Clinical Neuroendocrinology Branch National Institute of Mental Health NIH, Bethesda, MD 20892-1284, USA

\section{References}

1 Sandbrink R, Hartmann T, Masters CL, Beyreuther K. Genes contributing to Alzheimer's disease. Mol Psychiatry 1996; 1: 27-40.

2 Sandbrink R, Beyreuther K. Unraveling the molecular pathway of Alzheimer's disease: research about presenilins gathers momentum. Mol Psychiatry 1996; 1: 438-444.

3 Dean B, Crook JM, Opeskin K, Hill C, Keks N, Copolov $\mathrm{DL}$. The density of muscarinic $M_{1}$ receptors is decreased in the caudate-putamen of subjects with schizophrenia. Mol Psychiatry 1996; 1: 54-58.

4 Meador-Woodruff JH, King RE, Damask SP, Bovenkerk KA. Differential regulation of hippocampal AMPA and kainate receptor subunit expression by haloperidol and clozapine. Mol Psychiatry 1996; 1: 41-53.

5 Licinio J. Molecular Psychiatry: the integration of molecuIar medicineand clinical psychiatry. Mol Psychiatry 1996; 1: $1-3$.

6 Veldhuis JD. Editorial: Returning to the roots of endocrinology: the challenge of evaluating in vivo glandular secretory activity. Endocrinology 1990; 127: 2611-2617.

7 Veldhuis JD. Novel modalities for appraising individual and coordinate pulsatile hormone secretion: the paradigm of luteinizing and testosterone release in the aging male. Mol Psychiatry 1997; 2: 70-80. 